\section{Prime Numbers and the Riemann Hypothesis} Barry Mazur and William Stein

Cambridge University Press; 1st edition (April 11, 2016)

150 pages, $\$ 20.49$

ISBN-13: 978-1-1074-9943-0

\section{Introduction}

The centenary of Hilbert's problems and the announcement of the Clay Institute's millennial prize problems resulted in a number of very good trade books about the Riemann Hypothesis. In their book Prime Numbers and the Riemann Hypothesis, Barry Mazur and William Stein set themselves [p. vii] a different goal:

A reader of these books will get a fairly rich picture of the personalities engaged in the pursuit [of the Riemann Hypothesis], and of related mathematical and historical issues. That is not the mission of [this book]. We aim-instead-to explain, in a manner as direct as possible and with the least mathematical background required, what this problem is all about and why it is so important.

Mazur and Stein are distinguished mathematicians who have made significant contributions in areas the Riemann Hypothesis touches. Both care deeply about teaching. Mazur writes like an angel, and Stein is the

Donal O'Shea is professor of mathematics and president of New College of Florida. His email address is doshea@ncf. edu.

For permission to reprint this article, please contact:

reprint-permission@ams.org.

DOI: http://dx.doi.org/10.1090/noti1700 founder of SageMath, a powerful open-source mathematical software system, and CoCalc. So, if anyone could write an account of this sort, it is they. Nonetheless, the goal they set themselves is audacious and highly nontrivial. Even today, Riemann's paper is tough going for those without specialized knowledge in the area. Writing mathematically meaningful, but readily comprehensible, text about a highly technical subject for serious readers is an undertaking not to be underestimated. When that subject lies deep enough to have consequences in many other areas, that difficulty is compounded.

Mazur and Stein succeed brilliantly. The first half of their book assumes no knowledge of calculus, the next third requires some knowledge of real differential calculus, and the last sixth some complex analysis. I cannot think of an interested reader who will not profit from this little book. It will fascinate anyone who enjoys mathematics. It provides amateurs and students with an attractive and accessible entry to a compelling, but difficult realm of mathematics. Professional mathematicians and mathematical scientists will love it-so will electrical engineers and computer scientists. It deserves to become a classic.

\section{Riemann and His Hypothesis}

In 1859 at age thirty-two, Bernhard Riemann was appointed to the chair at the University of Göttingen previously held by his teachers Gauss and Dirichlet. The death of Dirichlet, to whom Riemann was very close, had left the chair vacant. For Riemann, the appointment would have been bittersweet. He desperately needed the income and recognition, but Dirichlet was one of his closest friends, and one of the few who understood him and his mathematics. Shortly afterwards the Prussian Academy of Sciences in Berlin elected Riemann as a corresponding member, and Kronecker encouraged 
him to submit a paper describing his research. Whether by design or serendipity, Riemann had been exploring some ideas that had intensely interested Gauss and Dirichlet. The resulting hastily-written, eight-page paper introduced the complex-valued function now known as the Riemann zeta function and related it to the number $\pi(X)$ of prime numbers less than or equal to a given quantity $X$. It introduced a host of astonishing new ideas and techniques. As Selberg [2] points out, the paper was clearly a research note sketching some ideas to which Riemann intended to return. There were holes that needed to be filled and Riemann's mathematical notebooks subsequently made it clear that Riemann had more material to present. Riemann would die seven years later, without returning to the subject. At the time, the paper had little discernible impact, but in time it would completely transform analytic number theory and reverberate across mathematics.

Thirty-five years later, Riemann's paper was better understood. The Prime Number Theorem, originally conjectured by Gauss and equivalent to the statement that no zeroes of the zeta function have real part equal to 1 , was established independently by Hadamard and de la Vallée Poussin in 1896. But a statement, now known as the Riemann Hypothesis, that Riemann conjectured would give much better information, remains unsolved to this day. Hilbert included it as the eighth problem among his list of twenty-three problems for the twentieth century. After giving a nod to then-recent progress on the distribution of prime numbers, he writes [2, p. 456]

It still remains to prove the correctness of an exceedingly important statement of Riemann, viz., that the zero points of the function $\zeta(s)$ defined by the series

$$
\zeta(s)=1+\frac{1}{2^{s}}+\frac{1}{3^{s}}+\frac{1}{4^{s}}+\ldots
$$

all have the real part $\frac{1}{2}$, except the well-known negative integral zeros. As soon as this proof has been successfully established, the next problem would consist in testing more exactly Riemann's infinite series for the number of primes below a given number and, especially, to decide whether the difference between the number of primes below a number $x$ and the integral logarithm of $x$ does in fact become infinite of an order not greater than $\frac{1}{2}$ in $x$. Furthermore, we should determine whether the occasional condensation of prime numbers which has been noticed in counting primes is really due to those terms in Riemann's formula which depend upon the first complex zeroes of the function $\zeta(s)$.

In the late 1990s, the Clay Mathematical Institute surveyed a representative group of mathematicians as to what they considered the most pressing open problems for the new millennium. There were, of course, differences of opinion, but all approached included two problems on their lists, the Poincaré Conjecture (now solved) and the Riemann Hypothesis. Both were among the seven "millennial" problems for the solution of each of which the Institute offered a one million dollar prize.

\section{The Book}

The authors begin by sorting their readership into three groups: 1) interested individuals who may not have had a calculus course, but who are comfortable with the notion of a graph of a function; 2) interested individuals who know some differential calculus; 3) interested individuals who know a little complex analysis. They then order their material accordingly. Part I, about seventy pages of the book, addresses the first group. Parts II and III, about forty pages in total, address the second group. Part IV, the shortest at twenty-five pages, addresses the third group, as do the ten pages of endnotes (which are terrific).

The first part opens with a careful discussion of primes, sieves, the frequency of primes, and the behavior of $\pi(X)$ as $X$ grows larger. The prose is spare, but engaging and informal. In addition to photographs of individuals (regrettably all male, all but one white), and excerpts from notebooks (see Figure 3), there are many tables and lots of well-chosen graphs (most generated by SageMath, with code available online). The function $\operatorname{Li}(X)$ is simply defined as the area from 2 to $X$ under the graph of $1 / \log (x)$. No fuss, no over-explanation, no integral signs. The authors zoom out of the graph of the step function $\pi(X)$, looking at it over larger and larger intervals. The reader clearly sees the graph that becomes a smooth curve at the resolution of the human eye and that the graph of $\frac{X}{\log (X)}$ appears to diverge from $\pi(X)$ faster than $\operatorname{Li}(X)$.

Tables are used to show the distinction between going to infinity at the same rate (as many leading digits as desired can be made to coincide) and to within square root error (first half of leading digits coincide). A wonderful discussion that links square root error to random walks follows. This is done by simulating and plotting lots of random walks on the line (see Figure 1), and it firmly establishes that square root error is the gold standard: the best that one can expect.

The authors present their first formulation of the Riemann Hypothesis as the statement that Gauss's logarithmic integral $\mathrm{Li}(X)$ is a square-root-close approximation to $\pi(X)$. This, of course, is the second part of Hilbert's statement of the Riemann Hypothesis, and their discussion allows the reader to appreciate the contrast between the Prime Number Theorem and the Riemann Hypothesis.

From here, the account pivots to a discussion of the in formation contained in $\pi(X)$, and in the graphs of related functions that contain the same information, which the authors refer to as carpentry on the staircase of primes. In particular, the authors consider the step function $\psi(X)$ that increases not just at prime numbers by 1 , but at all prime powers by the logarithm of the prime, and provide a second restatement of the Riemann Hypothesis as the assertion that $\psi(X)$ is square-root-close to $y=X$, the graph of which is the straight line in the first quadrant at 45 degrees off the $X$-axis.

The discussion about preservation of information and the utility of different representations of functions leads 

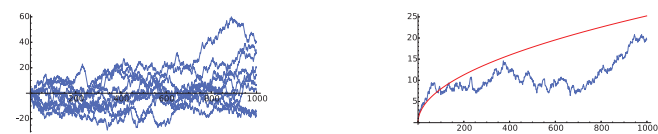

(A) Ten random walks

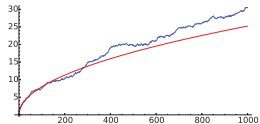

(B) One hundred

random walks
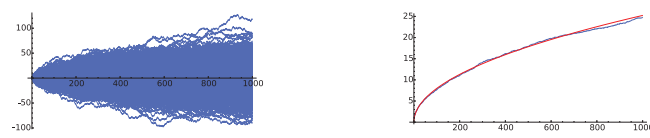

(C) One thousand

random walks

Figure 1. A wonderful discussion links square root error to random walks on the line. The figures on the left plot random walks starting at the origin; the blue curves on the right plot the average distance from the origin after $X$ steps of the random walks on the left, and the red line is the graph of $\sqrt{\frac{2}{\pi}} \cdot \sqrt{X}$.

naturally to a discussion of how the spectrum (that is, the collection of frequencies), amplitudes, and phases of a trigonometric sum more efficiently encode the information in the function than does sampling points on its graph. These discussions lead in turn, and seemingly ineluctably, to the notion of capturing the information in the graph of $\pi(X)$ by using something akin to Fourier analysis.

So ends Part I. It tells a complete and satisfying story, and a reader with no knowledge of calculus who leaves off at this point will have learned a great deal.

Parts II and III are aimed at readers with some, but minimal, differential calculus, and continue the information theme. The authors use the same pedagogical techniques as in the first section (many of which are nowadays used in reform calculus texts). They begin with a brief introduction to generalized functions, starting with the Dirac delta function, introduced as the limit of the derivatives of bump functions approximating a step function with one jump discontinuity. Since they assume a little familiarity with calculus, they are able to be precise about convergence. They discuss Fourier transforms of distributions with discrete support, transforms of trigonometric sums, and spike values. All this is done with lots of graphs.

With this in hand, they move to two trigonometric series that are related to one another in that the frequencies (the logarithms of prime powers) of the first are the spike values (the points of divergence) of the second, and vice versa the spike values of the first are the frequencies (or "prime spectrum") of the other. The first infinite sum comes right out of information in $\pi(X)$, and the graphs of the trigonometric sums approximating the series are computed, so the reader can see the graphs of these sums beginning to spike at the spike values.

There are many details here, and one of the delightful things is that the authors guide readers through the thicket, pointing out the essentials, and indicating what is central and what are conveniences. The calculus that the authors ask of their readers is not a facility with computation, but that the reader be comfortable with the key ideas of derivative and definite integral. For readers who have forgotten most of their calculus, the book will underscore the utility of those key concepts. There are many references to the literature and to sites where computations of the spectrum have been done, and I look forward to returning to the book to follow up on these.

The authors had clearly intended [p. 113] to provide a third restatement of the Riemann Hypothesis in terms of the concepts they developed in Parts II and III, but somewhere in the course of the many revisions of the book, this restatement went missing. Although this tacitly leaves an exercise for the reader, the omission is unfortunate as the restatement would have given the reader a sense of closure.

The book closes with Part IV, a beautiful short section, aimed at the reader with some knowledge of complex analysis. The authors return to Riemann's paper and present Riemann's formula for $\pi(X)$. Here again, the authors plot the graphs of the function $R(X)$ that Riemann used (see Figure 2), and the reader sees how much better Riemann's successive approximations are to $\pi(X)$ than $\operatorname{Li}(X)$.

The authors [p. 124] make the crucial point that the error terms can be explicitly computed once one knows the zeroes of the zeta function, and provide the "fourth" and final restatement of the Riemann Hypothesis as the conjecture that all non-trivial zeroes of the zeta function have imaginary part equal to $\frac{1}{2}$. This, of course, is the famous version that Hilbert states, and with which we are all familiar. The authors go on to write:

That a simple geometric property of these zeroes (lying on a line!) is directly equivalent to such profound (and more difficult to express) regularities among prime numbers suggests that these 

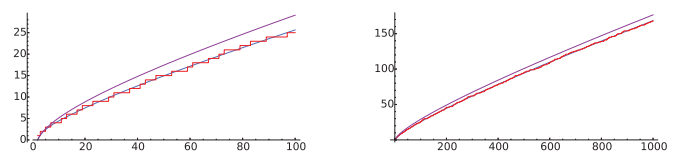

(A) Comparisons of $\operatorname{Li}(X)$ (top), $\pi(X)$ (middle), and $R(X)$ (bottom, computed using 100 terms on the left and 1000 terms on the right).

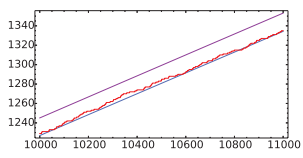

(A)

(B) Closeup comparison of $\operatorname{Li}(X)$ (top), $\pi(X)$ (middle), and $R(X)$ (bottom, computed using 11000 terms).

Figure 2. Riemann's initial approximation to $\pi(X)$ is much better than $\operatorname{Li}(X)$.

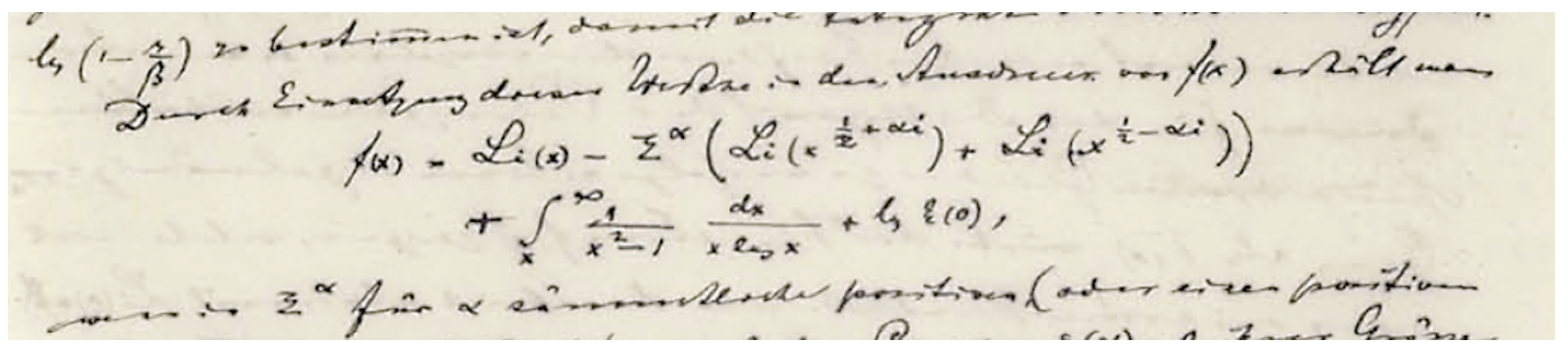

Figure 3. Riemann's exact formula for $\pi(X)$.

zeroes and the parade of Riemann's corrections governed by them-when we truly comprehend their message-may have lots more to teach us, may eventually allow us a more powerful understanding of arithmetic. ... Are the primes themselves no more than an epiphenomenon, behind which there lies, still veiled from us, a yet-to-be-discovered, yet-to-be-hypothesized, profound conceptual key to their perplexing orneriness?

The final chapter in Part IV exemplifies what is so special about the book. The authors want the reader to understand that the Riemann zeta function is one of a family of functions that arise in many other contexts. In these last few pages of the book, they could have been forgiven for going wild and citing loads of interesting, inscrutable examples. They do not. Rather, they concentrate on a single example that their hypothetical reader will understand, the distribution of Gaussian primes. They do not take shortcuts. They define norms, Gaussian integers and units, and then Gaussian primes. They plot the distributions of Gaussian primes $a+b i$ in the first quadrant of the plane with $a, b \leq 10$, and then $a, b \leq 100$. They then look at the staircase of primes (the graph of the number of Gaussian primes with norm less than or equal to $X$ ), and produce the graphs for norms up to 10 , $100,1000,10000$. In the process of zooming out, one sees the graphs approximating a smooth curve, and one has no difficulty believing their assertion that there is an analogue of the zeta function in this context. Only then do they mention that there is a larger story, the Grand Riemann Hypothesis, and give the example where zeta-type functions count solutions of polynomials of several variables in finite fields of characteristic $p$.

Seldom have I, as a reader, felt so respected. This is superb teaching, and beautiful writing.

\section{Conclusion}

The authors say that they wrote the book over a period of ten years, getting together for one week during the summers. Nonetheless, the book itself is highly disciplined. It is short and very attractively laid out. The four parts consist of a number of short chapters, which are perfect for sporadic reading.

The authors have given the mathematical community a great gift. Buy the book for yourselves, your friends, and your students. 


\section{References}

[1] DAvid, Hilbert, Mathematical Problems, Lecture delivered before the International Congress of Mathematicians at Paris in 1900. Translated by Mary Winston Newson. Bull. Amer. Math. Soc. 8(1902), pp. 437-479. The original appeared in Göttinger Nachrichten (1900) pp. 253-297.

[2] AtLe Selberg, The History of the Prime Number Theorem, The opening lecture at "In Celebration of the Centenary of the Proof of the Prime Number Theorem, A Symposium on the Riemann Hypothesis," Seattle, Washington, August 12-15, 1996, Sponsored by the American Institute of Mathematics. Handwritten slides available at publications.ias.edu/se1berg/section/2492.

\section{Image Credits}

Figures 1 and 2 courtesy of Barry Mazur and William Stein, Prime Numbers and the Riemann Hypothesis, 2016, Cambridge University Press, used with permission.

Figure 3 courtesy of SUB Göttingen, Cod. Ms. B. Riemann 3 : folio $18 \mathrm{r}$.

Author photo by Kim McDonald, New College of Florida.

\section{ABOUT THE AUTHOR}

Donal O'Shea came to New College after thirty-two years at Mount Holyoke College. He is author or co-author of a number of books including The Poincare Conjecture; Ideals, Varieties and Algorithms; Using Algebraic Geometry; Calculus in Context; and Laboratories in Mathematical Experimentation.

\section{FEATURED TITLES FROM THE}

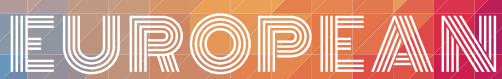

IIIIIA

50(C)IET VI

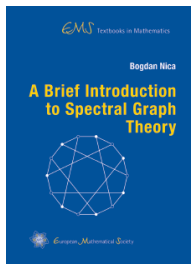

A Brief Introduction to Spectral Graph Theory

Bogdan Nica, McGill University, Montreal, Canada

Spectral graph theory starts by associating matrices to graphs-notably, the adjacency matrix and the Laplacian matrix. The general theme is then, first, to compute or estimate the eigenvalues of such matrices, and, second, to relate the eigenvalues to structural properties of graphs. As it turns out, the spectral perspective is a powerful tool. Some of its loveliest applications concern facts that are, in principle, purely graph theoretic or combinatorial. This text is an introduction to spectral graph theory, but it could also be seen as an invitation to algebraic graph theory.

EMS Textbooks in Mathematics, Volume 21; 2018; 168 pages; Hardcover; ISBN: 978-3-03719-188-0; List US\$48; AMS members US\$38.40; Order code EMSTEXT/21

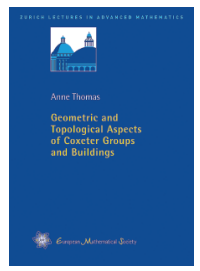

Geometric and Topological Aspects of Coxeter Groups and Buildings

Anne Thomas, University of Sydney, Australia

Coxeter groups are groups generated by reflections. They appear throughout mathematics. Tits developed the general theory of Coxeter groups in order to develop the theory of buildings. Buildings have interrelated algebraic, combinatorial and geometric structures and are powerful tools for understanding the groups which act on them. These notes focus on the geometry and topology of Coxeter groups and buildings, especially nonspherical cases. The emphasis is on geometric intuition, and there are many examples and illustrations.

Zurich Lectures in Advanced Mathematics, Volume 24; 2018; 160 pages; Softcover; ISBN: 978-3-03719-189-7; List US\$39; AMS members US\$31.20; Order code EMSZLEC/24

\section{Explore more titles at} bookstore.ams.org.

Publications of the European Mathematical Society (EMS). Distributed within the Americas by the American Mathematical Society.

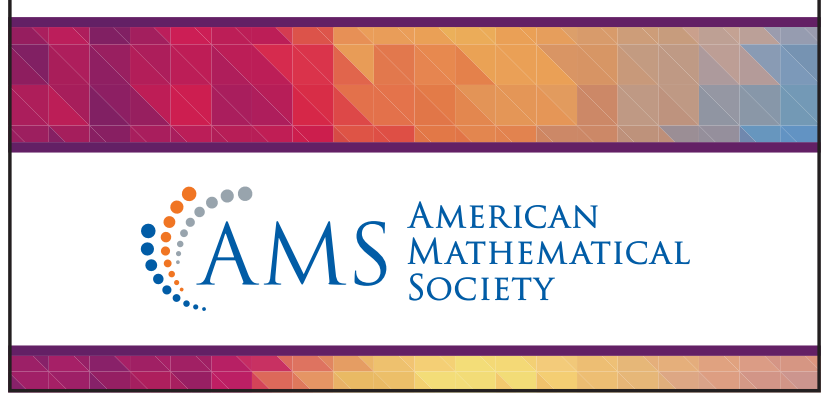

